\title{
THE CHAIN RECURRENT SET FOR MAPS OF THE CIRCLE
}

\author{
LOUIS BLOCK AND JOHN E. FRANKE
}

\begin{abstract}
For a continuous map of the circle to itself we give necessary and sufficient conditions for the chain recurrent set to be precisely the set of periodic points. We also examine the possible types of examples which can occur, where the set of periodic points is closed and nonempty, but there are nonperiodic, chain recurrent points.
\end{abstract}

1. Introduction. In studying the dynamics of a map $f$ from a space to itself, invariant sets, whose orbits satisfy some type of recurrence property, play an important role. Three such sets are the set of periodic points, the nonwandering set and the chain recurrent set, which we will denote respectively by $P(f), \Omega(f)$ and $R(f)$. In general, $P(f) \subset \Omega(f) \subset R(f)$, but equality need not hold. An obvious necessary condition for $P(f)=\Omega(f)$ or $P(f)=R(f)$ is that $P(f)$ must be closed. For maps of the interval this condition is sufficient to guarantee that $P(f)=\Omega(f)=R(f)$ $[\mathbf{2}, \mathbf{4}, \mathbf{8}, \mathbf{1 0}, \mathbf{1 4}]$. For maps of the circle a necessary and sufficient condition for $P(f)=\Omega(f)$ is that $P(f)$ must be closed and nonempty $[\mathbf{1}]$. In this paper we give necessary and sufficient conditions for $P(f)=R(f)$ for maps of the circle.

To give these conditions we must introduce the concept of a generalized attracting neighborhood. Let $f$ be a continuous map of the circle to itself and let $p$ be a periodic point of $f$ of (least) period $n$. A generalized attracting neighborhood of $p$ is an open neighborhood $V_{p}$ of $p$ with $\bar{V}_{p} \neq S^{1}$ and $f^{n}\left(\bar{V}_{p}\right) \subset V_{p}$. It is important in the definition that $V_{p}$ is open and its closure is mapped inside $V_{p}$ by $f^{n}$. For example, let $A$ be any closed subset of $[0,1]$ with $0 \in A$ and $1 \in A$. There is a homeomorphism $g$ of $[0,1]$ onto itself with $g(x)=x$ if $x \in A$ and $g(x)<x$ if $x \notin A$. Let $f$ be the homeomorphism of the circle, $S^{1}$, onto itself obtained from $g$ by identifying 0 and 1 . The reader should verify that no fixed point of $f$ has a generalized attracting neighborhood. Note also that in this example $P(f)$ is the nonempty closed set $A$, while $R(f)=S^{1}$.

On the other hand, let $g$ be a homeomorphism of $[0,1]$ onto itself which has six fixed points $0, \frac{1}{5}, \frac{2}{5}, \frac{3}{5}, \frac{4}{5}$ and 1 with $g(x)>x$ if $x \in\left(0, \frac{1}{5}\right) \cup\left(\frac{2}{5}, \frac{3}{5}\right) \cup\left(\frac{3}{5}, \frac{4}{5}\right)$ and $g(x)<x$ if $x \in\left(\frac{1}{5}, \frac{2}{5}\right) \cup\left(\frac{4}{5}, 1\right)$. The reader should verify that if $f$ is the homeomorphism of the circle to itself obtained from $g$ then each of the five fixed points of $f$ has a generalized attracting neighborhood. Thus, while a sink (or contracting periodic point) always has a generalized attracting neighborhood, a source (or expanding periodic point) or a nonhyperbolic periodic point may also have a generalized attracting neighborhood.

Received by the editors November 29, 1982 and, in revised form, January 12, 1984.

1980 Mathematics Subject Classification. Primary 54H20. 
THEOREM A. Let $f$ be a continuous map of the circle to itself. $R(f)=P(f)$ if and only if $P(f)$ is closed and nonempty and for every $x \in S^{1} \backslash P(f)$, some element of $\omega(x)$ has a generalized attracting neighborhood.

In Theorem $\mathrm{A}, \omega(x)$ denotes the set of limit points of the orbit of $x$. Note that if $P(f)$ is closed and nonempty, $\Omega(f)=P(f)[\mathbf{1}]$, and hence any element of $\omega(x)$ is a periodic point. The proof of Theorem A shows that if $R(f)=P(f)$ and $x \in S^{1} \backslash P(f)$, then every element of $\omega(x)$ has a generalized attracting neighborhood.

We remark that Theorem $\mathrm{A}$ is similar in flavor to results in other settings which give necessary and sufficient conditions for the nonwandering set to equal the chain recurrent set (see $[\mathbf{1 2}$, Theorem $3.11 ; \mathbf{3}, \mathbf{5}, \mathbf{7}, \mathbf{9}, \mathbf{1 3}]$ ). However, one cannot extend Theorem A to show that $\Omega(f)=R(f)$ if and only if for every $x \in S^{1} \backslash \Omega(f)$ some element of $\omega(x)$ has a generalized attracting neighborhood. The "if" part of this statement would imply that $\Omega(f)=R(f)$ for any map of an interval to itself (since any map of an interval may be extended to a map of the circle which is not onto), but $\Omega(f)$ need not equal $R(f)$ for maps of the interval, as an example in [2] shows.

The remainder of the paper is concerned with the (somewhat technical) problem of determining what types of examples are possible of maps $f$ of the circle with $P(f)$ closed and nonempty but $P(f) \neq R(f)$. Such a map $f$ must have a nonperiodic point $x$ such that all elements of $\omega(x)$ are perodic but do not have generalized attracting neighborhoods. Clearly this can occur if $f$ has a nonhyperbolic periodic point which does not have a generalized attracting neighborhood as in the first example above. Thus, we restrict our attention to the case where any nonhyperbolic periodic point has a generalized attracting neighborhood, i.e. where any periodic point is either expanding (see $\S 2$ for definition) or has a generalized attracting neighborhood. An example in this case would require a point $x$ such that all elements of $\omega(x)$ are expanding periodic points. The next theorem shows that this is impossible if the orbit of $x$ is infinite.

THEOREM B. Suppose that the orbit of a point $x$ (under a continuous map of the circle to itself) is infinite. Then some element of $\omega(x)$ is not an expanding periodic point.

One can construct examples where the orbit of $x$ is finite and all elements of $\omega(x)$ are expanding periodic points (i.e. $x$ is eventually an expanding periodic point) which do not have generalized attracting neighborhoods. For example, let $g$ be the map of $[0,1]$ to itself with $g(0)=0, g\left(\frac{1}{4}\right)=1, g\left(\frac{1}{2}\right)=\frac{9}{16}, g\left(\frac{3}{4}\right)=\frac{11}{16}$ and $g(1)=1$, such that $g$ is linear on each of the intervals $\left[0, \frac{1}{4}\right],\left[\frac{1}{4}, \frac{1}{2}\right],\left[\frac{1}{2}, \frac{3}{4}\right]$ and $\left[\frac{3}{4}, 1\right]$. Let $f$ be the map of the circle to itself obtained from $g$. Then $\Omega(f)$ consists of the expanding fixed point, 0 , and the contracting fixed point, $\frac{5}{8}$, but the point $\frac{1}{4}$ is chain recurrent as are its inverse images.

The previous example yields a continuous map $f$ of the circle to itself with $P(f)$ closed and nonempty such that any periodic point is either expanding or has a generalized attracting neighborhood, but $R(f) \neq P(f)$. Note that in the example there is a critical point $x$ (see $\S 2$ for definition) such that for some positive integer $k, f^{k}(x)$ is an expanding periodic point which has no generalized attracting neighborhood (i.e. $x=\frac{1}{4}$ and $k=1$ ). Our final theorem shows that this must occur in any such example. 
THEOREM C. Let $f$ be a continuous map of the circle to itself. Suppose that $P(f)$ is closed and nonempty and that any periodic point of $f$ is either expanding or has a generalized attracting neighborhood. Suppose also that for any critical point $x$ of $f$, if $f^{k}(x)$ is periodic for some positive integer $k$, then $f^{k}(x)$ has a generalized attracting neighborhood. Then $R(f)=P(f)$.

2. Background. Let $f$ be a continuous map from a compact metric space $(X, d)$ into itself. An $\varepsilon$-chain from $x$ to $y$ (where $x, y \in X$ ) is a finite sequence of points $\left\{x_{0}, x_{1}, \ldots, x_{n}\right\}$ of $X$ with $x=x_{0}, y=x_{n}$ and $d\left(f\left(x_{i-1}\right), x_{i}\right)<\varepsilon$ for $i=1, \ldots, n$. We say $x$ can be chained to $y$ if for every $\varepsilon>0$ there is an $\varepsilon$-chain from $x$ to $y$, and we say $x$ is chain recurrent if $x$ can be chained to $x$. The set of all chain recurrent points is denoted by $R(f)$.

A subset $Y$ of $X$ is called positively chain invariant if for every $y \in Y$ and $x \in X \backslash Y, y$ cannot be chained to $x$. Proofs of the following two lemmas may be found in $[\mathbf{2}]$. The first lemma was also noted in [11].

LEMMA 1. $R(f)=R\left(f^{n}\right)$ for any positive integer $n$.

LEMMA 2. Let $Y$ be a positively chain invariant subset of $X$. If $x \notin Y$ and $f^{k}(x) \in Y$ for some positive integer $k$, then $x \notin R(f)$.

If $Y \subset X$ we let $f \mid Y$ denote the restriction of $f$ to $Y$ and $\bar{Y}$ denote the closure of $Y$.

LEMMA 3. If $Y$ is an open subset of $X$ such that $f(\bar{Y}) \subset Y$ then $\bar{Y}$ is positively chain invariant and $R(f) \cap \bar{Y}=R(f \mid \bar{Y})$.

Proof. Let $\varepsilon_{1}=d(X \backslash Y, f(\bar{Y}))$. Note that $\varepsilon_{1}>0$ since $X \backslash Y$ and $f(\bar{Y})$ are disjoint compact sets. If $y \in \bar{Y}$ and $0<\varepsilon<\varepsilon_{1}$, then every $\varepsilon$-chain starting at $y$ is completely contained in $\bar{Y}$. Thus $\bar{Y}$ is positively chain invariant and $R(f) \cap \bar{Y}=$ $R(f \mid \bar{Y})$. Q.E.D.

The following lemma, which slightly relaxes the hypothesis of the previous lemma in the case where $Y$ is an open interval, is proved as in Lemma 4 of [2]. The lemma allows for the case where one endpoint of $Y$ is mapped to the other.

LEMMA 4. Let $f$ be a continuous map of $S^{1}$ to itself. Let $Y$ be a proper open subinterval of $S^{1}$ with $f(\bar{Y}) \subset \bar{Y}$. If $a$ and $b$ are the endpoints of $Y$ and $f(a) \neq a$ while $f(b) \in Y$, then $\bar{Y}$ is positively chain invariant.

For the rest of the paper, $f$ will denote a continuous map of the circle to itself and $d$ will denote a metric on the circle. A point $x \in S^{1}$ is called a critical point of $f$ if $f$ fails to be injective on every neighborhood of $x$.

Let $p$ be a periodic point of $f$ of (least) period $n$ which is not a critical point of $f^{n}$. Let $k=n$ if $f^{n}$ preserves orientation at $p$, and $k=2 n$ if $f^{n}$ reverses orientation at $p$. We call $p$ an expanding periodic point if there is an open neighborhood $V_{p}$ of $p$ such that for each $x \in V_{p}, d\left(f^{k}(x), p\right)>d(x, p)$.

If $p$ is a fixed point of $f$, its unstable manifold is defined to be

$$
W^{u}(p, f)=\bigcap\left(\bigcup_{n=0}^{\infty} f^{n}(V)\right)
$$


where the intersection is taken over all neighborhoods $V$ of $p$. If $p$ is an expanding fixed point then $W^{u}(p, f)$ is an invariant interval with $p$ as an interior point. We will use the following lemma in the proof of Theorem $\mathrm{C}$.

LEMMA 5. Let $f$ be a continuous map of the circle to itself and let $p$ be an expanding fixed point of $f$. Let $q$ be an endpoint of $W^{u}(p, f)$.

(1) If $q \in W^{u}(p, f)$, then $q$ is in the orbit of a critical point of $f$.

(2) If $q \notin W^{u}(p, f)$, then $q$ is not expanding and is either a fixed point of $f$ or a point of period two.

PROOF. There is an open neighborhood $V$ of $p$ with $W^{u}(p, f)=\bigcup_{n=0}^{\infty} f^{n}(V)$.

If $q \in W^{u}(p, f)$ there is a point $r \in V$ and a positive integer $n$ such that $f^{n}(r)=q$. One of the points $r, f(r), \ldots, f^{n-1}(r)$ must be a critical point, or else $f^{r}(V)$ would contain a neighborhood of $q$ and $q$ would not be an endpoint of $W^{u}(p, f)$. This proves (1).

Now suppose that $q \notin W^{u}(p, f)$. Since

$$
\begin{aligned}
& f\left(W^{u}(p, f)\right)=W^{u}(p, f) \quad \text { and } \\
& f\left(\overline{W^{u}(p, f)}\right)=\overline{W^{u}(p, f)},
\end{aligned}
$$

$q$ is either a fixed point of $f$ or a point of period two. In the latter case, we may replace $f$ by $f^{2}$, so we assume that $q$ is a fixed point of $f$.

Let $\overline{W^{u}(p, f)}=\left[q_{1}, q\right]$. Note that $f\left(q_{1}\right) \neq q$ (because if $f\left(q_{1}\right)=q$ then $q_{1} \in$ $W^{u}(p, f)$ and hence $\left.q \in W^{u}(p, f)\right)$.

Suppose $q$ is an expanding fixed point of $f$. Since $q$ is an endpoint of $W^{u}(p, f)$, $f$ preserves orientation at $q$. Let $V_{q}=(a, b)$ be a neighborhood of $q$ such that $d(f(x), q)>d(x, q)$ for all $x \in V_{q}$, and $f(x) \in(p, q)$ for all $x \in(a, q)$. Then $f\left(\left[q_{1}, a\right]\right)=\left[q_{1}, c\right]$, where $c \in(a, q)$, and $f\left(\left[q_{1}, c\right]\right) \subset\left[q_{1}, c\right]$. This implies that $q \notin \overline{W^{u}(p, f)}$, a contradiction. This proves (2). Q.E.D.

We conclude this section with a lemma which shows that if one point in a periodic orbit has a generalized attracting neighborhood then the others do also.

LEMMA 6. Let $y$ be a periodic point of a continuous map $f$ of the circle to itself. If $y$ has a generalized attracting neighborhood then so does each point in the orbit of $y$.

PROOF. Let $n$ denote the period of $y$ and let $y_{n}=f^{n-1}(y)$. It suffices to show that $y_{n}$ has a generalized attracting neighborhood.

Let $V_{1}$ be a connected generalized attracting neighborhood of $y$. Let $V_{n}$ denote the component of $f^{-1}\left(V_{1}\right)$, which contains $y_{n}$. Then $V_{n}$ is an open interval and $f\left(\bar{V}_{n}\right) \subset \bar{V}_{1}$.

If $\bar{V}_{n}=S^{1}$, then $f$ is not onto and any periodic point has a generalized attracting neighborhood. Thus, we may assume that $\bar{V}_{n} \neq S^{1}$. It follows that $\bar{V}_{n}$ is a proper closed interval $[a, b]$ on $S^{1}$ with $f(a) \notin V_{1}$ and $f(b) \notin V_{1}$. Hence, $a \notin f^{n-1}\left(\bar{V}_{1}\right)$ and $b \notin f^{n-1}\left(\bar{V}_{1}\right)$. Thus,

$$
f^{n}\left(\bar{V}_{n}\right)=f^{n-1}\left(f\left(\bar{V}_{n}\right)\right) \subset f^{n-1}\left(\bar{V}_{1}\right) \subset V_{n}
$$

so $V_{n}$ is a generalized attracting neighborhood of $y_{n}$. Q.E.D. 


\section{Proofs of the theorems.}

Proof of TheOrem A. First suppose $R(f)=P(f)$. Since $R(f)$ is closed and nonempty, so is $P(f)$. Let $x \in S^{1} \backslash P(f)$ and let $y \in \omega(x)$. Then $y \in \Omega(f)=P(f)$. We will show that $y$ has a generalized attracting neighborhood.

Let $R_{\varepsilon}(y)$ denote the set of $z \in S^{1}$ such that there is an $\varepsilon$-chain from $y$ to $z$. It follows from the definition of $\varepsilon$-chain that $R_{\varepsilon}(y)$ is open. Since $x \notin R(f)$, for some $\varepsilon>0, x \notin R_{\varepsilon}(y)$.

Let $V_{y}$ denote the component of $R_{\varepsilon}(y)$ that contains $y$. Then $V_{y}$ is an open interval, invariant under $f^{n}$, where $n$ is the period of $y$. In fact, since there is no $\varepsilon$-chain from $y$ to the endpoints of $V_{y}$, for any $z \in V_{y}$ the distance from $f^{n}(z)$ to any endpoint of $V_{y}$ is at least $\varepsilon$. Thus, $f^{n}\left(\bar{V}_{y}\right) \subset V_{y}$. If $f$ is onto then $\bar{V}_{y} \neq S^{1}$ and $V_{y}$ is a generalized attracting neighborhood of $y$, while if $f$ is not onto it follows easily that any periodic point of $f$ (and in particular $y$ ) has a generalized attracting neighborhood.

Now suppose that $P(f)$ is closed and nonempty and for every $x \in S^{1} \backslash P(f)$. some element of $\omega(x)$ has a generalized attracting neighborhood. Let $x \in S^{1} \backslash P(f)$. We will show that $x \notin R(f)$.

Let $y$ be an element of $\omega(x)$ which has a generalized attracting neighborhood and denote the orbit of $y$ by $\left\{y_{1}, y_{2}, \ldots, y_{n}\right\}$. By Lemma 6 , each $y_{k}$ has a generalized attracting neighborhood $V_{k}$. Since $f^{n} \mid \bar{V}_{k}$ is a map of a compact interval to itself, it follows from [2] that $R\left(f^{n} \mid \bar{V}_{k}\right)=P\left(f^{n} \mid \bar{V}_{k}\right)$. Thus, if $x \in \bar{V}_{k}$ for some $k$ then $x \notin R\left(f^{n} \mid \bar{V}_{k}\right)$. Hence, by Lemmas 1 and $3, x \notin R(f)$.

Thus, we may assume that $x \notin \bar{V}_{k}$ for each $k=1, \ldots, n$. Since $y \in \omega(x)$, for some $k=1, \ldots, n$ and some positive integer $j, f^{j n}(x) \in V_{k}$. By Lemmas 2 and 3 . $x \notin R\left(f^{n}\right)$, so by Lemma $1, x \notin R(f)$.

PROOF OF THEOREM B. Suppose each element of $\omega(x)$ is an expanding periodic point. Let $y_{1} \in \omega(x)$. Let $n_{1}=2 j_{1}$, where $j_{1}$ is the period of $y_{1}$. Let $\varepsilon_{1}$ denote the distance from $y_{1}$ to the set of periodic points $z$ of $f$ such that $z \neq y_{1}$ and the period of $z$ is at most $n_{1}$. Since $y_{1}$ is an expanding point, $\varepsilon_{1}>0$. There are neighborhoods $W_{1}$ and $V_{1}$ of $y_{1}$ such that $f^{n_{1}}$ is expanding on $V_{1}, f^{n_{1}}\left(W_{1}\right) \subset V_{1}$, and the diameter of $V_{1}$ is less than $\varepsilon_{1} / 3$. It follows that there is a point $y_{2}$ in $\omega(x) \cap\left(\bar{V}_{1} \backslash \bar{W}_{1}\right)$.

Let $O_{1}$ be the union of neighborhoods of diameter $\varepsilon_{1} / 3$ about each periodic point $z \neq y_{1}$ whose period is at most $n_{1}$ and let $K_{1}=S^{1} \backslash\left(O_{1} \cup W_{1}\right)$. Then $y_{2}$ is in the interior of $K_{1}$. Let $n_{2}=2 j_{2}$, where $j_{2}$ is the period of $y_{2}$, and note that $n_{2}>n_{1}$. Let $\varepsilon_{2}$ denote the distance from $y_{2}$ to the set of periodic points $z$ of $f$ such that $z \neq y_{2}$ and the period of $z$ is at most $n_{2}$. Let $O_{2}$ be the union of neighborhoods of diameter $\varepsilon_{2} / 3$ about each periodic point $z \neq y_{2}$ whose period $k$ satisfies $n_{1}<k \leq n_{2}$. Let $W_{2}$ and $V_{2}$ be neighborhoods of $y_{2}$ with $\bar{V}_{2}$ contained in the interior of $K_{1}$ such that $f^{n_{2}}$ is expanding on $V_{2}, f^{n_{2}}\left(W_{2}\right) \subset V_{2}$, and the diameter of $V_{2}$ is less than $\varepsilon_{2} / 3$. There is a point $y_{3}$ in $\omega(x) \cap\left(\bar{V}_{2} \backslash \bar{W}_{2}\right)$. Let

$$
K_{2}=S^{1} \backslash\left(O_{1} \cup W_{1} \cup O_{2} \cup W_{2}\right)
$$

and note that $y_{3}$ is in the interior of $K_{2}$.

Define $K_{n}$ inductively as above, and let $G_{n}=K_{n} \cap \omega(x)$. Then $\left\{G_{n}\right\}$ is a decreasing family of nonempty compact sets, so $\bigcap_{n=1}^{\infty} G_{n}$ is nonempty. Any point in the intersection is in $\omega(x)$ but is not periodic, a contradiction. 
Proof of Theorem C. Let $x \in S^{1}$ and suppose that $x$ is not periodic. We must show that $x$ is not chain recurrent. We remark that if any element $q$ of $\omega(x)$ has a generalized attracting neighborhood $V_{q}$, then $x$ is not chain recurrent. This follows as in the proof of Theorem A. Thus, we may assume that each element of $\omega(x)$ is an expanding periodic point. By Theorem $\mathrm{B}, x$ is eventually periodic.

By the remark above and Lemma 1, we may assume that for some positive integer $k, f^{k}(x)$ is an expanding fixed point which has no generalized attracting neighborhood. Then, by hypothesis, none of the points $x, f(x), \ldots, f^{k-1}(x)$ are critical points. This implies that $f^{k}$ maps an open interval about $x$ onto an open interval about $f^{k}(x)$. Thus, if $x \in \overline{W^{u}\left(f^{k}(x), f\right)}$, then $x \in \Omega(f)=P(f)$. Hence $x \notin \overline{W^{u}\left(f^{k}(x), f\right)}$.

Let $q_{1}$ and $q_{2}$ denote the (distinct) endpoints of $\overline{W^{u}\left(f^{k}(x), f\right)}$. We consider three cases.

First, suppose that neither $q_{1}$ nor $q_{2}$ is a fixed point of $f^{2}$. Then, by Lemma 4, $\overline{W^{u}\left(f^{k}(x), f\right)}$ is positively chain invariant. Since this set contains $f^{k}(x)$ but not $x$, $x$ is not chain recurrent by Lemma 2 .

Sccond, suppose that $q_{1}$ and $q_{2}$ are both fixed points of $f^{2}$. Replacing $f$ by $f^{2}$ if necessary, we may assume that $q_{1}$ and $q_{2}$ are both fixed points of $f$. By Lemma 5 and our hypothesis, $q_{1}$ and $q_{2}$ have generalized attracting neighborhoods $V_{q_{1}}$ and $V_{q_{2}}$. If $x \in \bar{V}_{q_{1}}$ or $x \in \bar{V}_{q_{2}}$, then $x$ is not chain recurrent by Lemma 3 and the theorem of $[\mathbf{2}]$. hence, we may assume that $x \notin\left(\bar{V}_{q_{1}} \cup \bar{V}_{q_{2}}\right)$. It follows from Lemma 3 that $\overline{W^{u}\left(f^{k}(x), f\right) \cup V_{q_{1}} \cup V_{q_{2}}}$ is positively chain invariant. Since this set contains $f^{k}(x)$ but not $x, x$ is not chain recurrent by Lemma 2 .

Finally, suppose that $f^{2}\left(q_{1}\right)=q_{1}$ and $f^{2}\left(q_{2}\right) \neq q_{2}$. If $f\left(q_{1}\right) \neq q_{1}$, it follows as in the first case that $x$ is not chain recurrent. If $f\left(q_{1}\right)=q_{1}$, then as in the second case $q_{1}$ has a generalized attracting neighborhood $V_{q_{1}}$ and $x$ is not chain recurrent.

\section{REFERENCES}

1. L. Block, E. Coven, I. Mulvey and Z. Nitecki, Homoclinic and nonwandering point for maps of the circle, Ergodic Theory Dynamical Systems (to appear).

2. L. Block and J. Franke, The chain recurrent set for maps of the interval, Proc. Amer. Math. Soc. 87 (1983), 723727.

3. C. Conley, Isolated invariant sets and the Morse index, CBMS Regional Conf. Ser. in Math., no. 38, Amer. Math. Soc., Providence, R.I., 1976.

4. V. V. Fedorenko and A. N. Sarkovskiı̌, Continuous maps of the interval with a closed set of periodic points, Studies of Differential and Differential-Delay Equations, Kiev, 1980, pp. 137145. (Russian)

5. J. Franke and J. Selgrade, Hyperbolicity and chain recurrence, J. Differential Equations 26 (1977), 2736.

6. Z. Nitecki, Differentiable dymamics, M.I.T. Press, Cambridge, Mass., 1971.

7. __ Explosions in completely unstable flows. I. preventing explosions, Trans. Amer. Math. Soc. 245 (1978), 4361.

8. __ Maps of the interval with closed period set, Proc. Amer. Math. Soc. 85 (1982), 451456.

9. Z. Nitecki and M. Shub, Filtrations, decompositions, and explosions, Amer. J. Math. 97 (1976), 10291047.

10. A. N. Sarkovskiı̌, On cycles and the structure of a continuous mapping, Ukrainian Math. J. 17 (1965), 104 111. (Ukrainian)

11. K. Sawada, On the iterations of diffeomorphisms without $C^{0}-\Omega$ explosions: an example, Proc. Amer. Math. Soc. 79 (1980), 110112. 
12. M. Shub, Stabilité global des systémes dymamiques, Asterisque, vol. 56, Soc. Math. France, Paris, 1978.

13. M. Shub and S. Smale, Beyond hyperbolicity, Ann. of Math. (2) 96 (1972), 587591.

14. J. C. Xiong, Continuous self-maps of the closed interval whose periodic points form a closed set. .J. China Univ. Sci. 11 (1981), 14-23.

Department of Mathematics, University of Florida, Gainesville, Florida 32611

Department of Mathematics, North Carolina State University, Raleigh. NORTH CAROLINA 27695-8205 